\title{
Polymer 2-Polymer 3 Interactions in Water 1/Polymer 2/Polymer 3 Ternary Systems
}

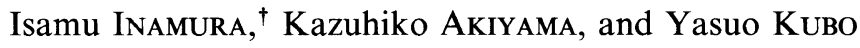 \\ Department of Material Science, Interdisciplinary Faculty of \\ Science and Engineering, Shimane University, Matsue 690, Japan.
}

(Received May 27, 1996)

\begin{abstract}
The polymer 2-polymer 3 interactions $\Delta b_{23}$, for water-poly(ethylene glycol) (PEG)-poly(vinyl alcohol) (PVA), water-poly( $N$-vinylpyrrolidone) (PVP)-PVA, water-dextran (Dex)-PVA, water-pullulan (Pul)-PVA, water-PVPPEG, water-Dex-PEG, water-Pul-PEG, water-PVP-Dex, water-Pul-PVP system were studied. For only the water-Dex-PVA system, $\Delta b_{23}$ is positive, but for the other systems negative. This indicates that most polymer pairs have repulsive interactions and are incompatible in water.
\end{abstract}

KEY WORDS Interaction / Polymer / Compatibility / Incompatibility /

Viscometry is a simple and reliable method to investigate interactions of macromolecules in solution. Using Huggins' plots, the polymer 2-polymer 3 interaction parameter, $\Delta b_{23}$ was introduced for solvent 1-polymer $2-$ polymer 3 ternary systems by the method of Krigbaum and Wall. ${ }^{1,2}$ According to Krigbaum and Wall, positive $\Delta b_{23}$ values indicate attractive interactions and compatibility, whereas negative values repulsive interactions and indicate incompatibility. Here, attractive, repulsive, compatible and incompatible have only relative meaning.

Recently, one of the authors obtained negative $\Delta b_{23}$ for water 1-polymer 2 (PVA)-polymer 3 (PEG), though the absolute values were small. ${ }^{3}$ Therefore, it can be inferred that weak repulsive interaction exists between PVA and PEG in water-PVA-PEG system. Suto et al. ${ }^{4}$ investigated the viscometric behavior of aqueous solutions of polymer mixtures involving a liquid crystalline polymer, and reported repulsive interactions between hydroxypropyl cellulose (liquid crystalline polymer) and hydroxyethyl cellulose.

In this paper, $\Delta b_{23}$ were determined for various polymer pairs in water, of poly(vinyl alcohol) (PVA), polyethylene glycol (PEG), poly( $N$-vinylpyrrolidone) (PVP), dextran (Dex), and pullulan (Pul), at various temperatures. $\Delta b_{23}$ for water-Dex-PVA was positive, and $\Delta b_{23}$ for the other systems were negative.

\section{EXPERIMENTAL}

\section{Materials}

The characterization of the water-soluble polymers are shown in Table I. PVA, PEG, and PVP were purified by reprecipitation in water-methanol, benzene-acetone, and water-acetone, respectively. Dex and Pul were used without further purification. The degree of saponification of PVA is $98.5 \mathrm{~mol} \%$.

\section{Measurement of Viscosity}

Ubbelohde viscometer was used to obtain viscosity. Measurement of viscosity was performed at 10, 20, 30, or $40^{\circ} \mathrm{C}$.

\footnotetext{
† To whom correspondence should be addressed
}

The Method of Calculation of $\Delta b_{23}$

Polymer 2-polymer 3 interaction parameter, $\Delta b_{23}$ was calculated as follows. ${ }^{2}$

$$
\frac{\left(\eta_{\mathrm{sp}}\right)_{\mathrm{m}}}{c_{\mathrm{m}}}=[\eta]_{\mathrm{m}}+b_{\mathrm{m}} c_{\mathrm{m}}
$$

$c_{\mathrm{m}}$ the total concentration of polymer, $c_{\mathrm{m}}=c_{2}+c_{3}$, and $[\eta]_{\mathrm{m}}$ the intrinsic viscosity of the mixture, theoretically defined as

$$
[\eta]_{\mathrm{m}}=[\eta]_{2} x_{2}+[\eta]_{3} x_{3}
$$

$x_{\mathrm{i}}$ is the weight fraction of polymer i. Equation 2 is only valid for non-interacting systems. $b_{\mathrm{m}}$ defines global interactions between all polymeric species

$$
b_{\mathrm{m}}=\left[\sum x_{\mathrm{i}}\left(b_{\mathrm{i}}\right)^{1 / 2}\right]^{2}=x_{2}{ }^{2} b_{22}+2 x_{2} x_{3} b_{23}+x_{3}{ }^{2} b_{33}
$$

interactions of the $b_{\mathrm{ii}}$ type may be expressed as

$$
b_{\mathrm{ii}}=k_{\mathrm{i}}^{\prime}[\eta]_{\mathrm{i}}^{2},
$$

$k^{\prime}$, the Huggins constant of the i component.

$b_{23}$ is a complex interaction parameter in which hydrodynamic and thermodynamic interactions as well as possible associations are included. Assuming the absence of thermodynamic interactions of $\mathrm{i}-\mathrm{i}$ and $\mathrm{j}-\mathrm{j}$ types in the corrresponding binary systems

$$
b_{23}^{*}=\left(b_{22} b_{33}\right)^{1 / 2} \text {, }
$$

where superscript * means "theoretical", $b_{23}$ may be obtained experimentally by insertion in eq 3 of the experimental value previously obtained from eq 1 .

$$
\Delta b_{23}=b_{23}-b_{23}^{*}
$$

Table I. Characterization of water-soluble polymers

\begin{tabular}{ccl}
\hline Polymer & Molecular weight $\left(M_{w}\right)$ & \multicolumn{1}{c}{ Supplier } \\
\hline PVA & 77000 & Kuraray Co., Ltd. \\
PEG & 20000 & Kishida Chemical Co., Ltd. \\
PVP & 40000 & Kishida Chemical Co., Ltd. \\
Dex & $60000-90000$ & Wako Pure Chemical Industries \\
Pul & 200000 & Sanwa Denpun Co., Ltd. \\
\hline
\end{tabular}




\section{RESULTS AND DISCUSSION}

Figure 1 shows $\eta_{\mathrm{sp}} / C$ vs. $C$ for dilute water-Dex-PVA system at $30^{\circ} \mathrm{C}$. All Huggins' plots gave straight lines. The intrinsic viscosity $[\eta]$ and Huggins' constant $k^{\prime}$ were evaluated from the intercept and slope of the plots, respectively. Other $[\eta]$ and $k^{\prime}$ were evaluated for other polymer pairs in water. Polymer composition dependence of $[\eta]$ and $k^{\prime}$ for all water 1-polymer 2-polymer 3 systems were obtained. [ $\eta]$ and $k^{\prime}$, and polymer composition dependence are not shown here. The additivity of $[\eta]$ was obtained in all systems with respect to polymer composition. As the temperature increased, $[\eta]$ decreased for all systems. Therefore, as temperature increased, the random coil of the polymer shrank. $k^{\prime}$ for all systems gave the curves, and increased with temperature. Therefore, as the temperature increased,

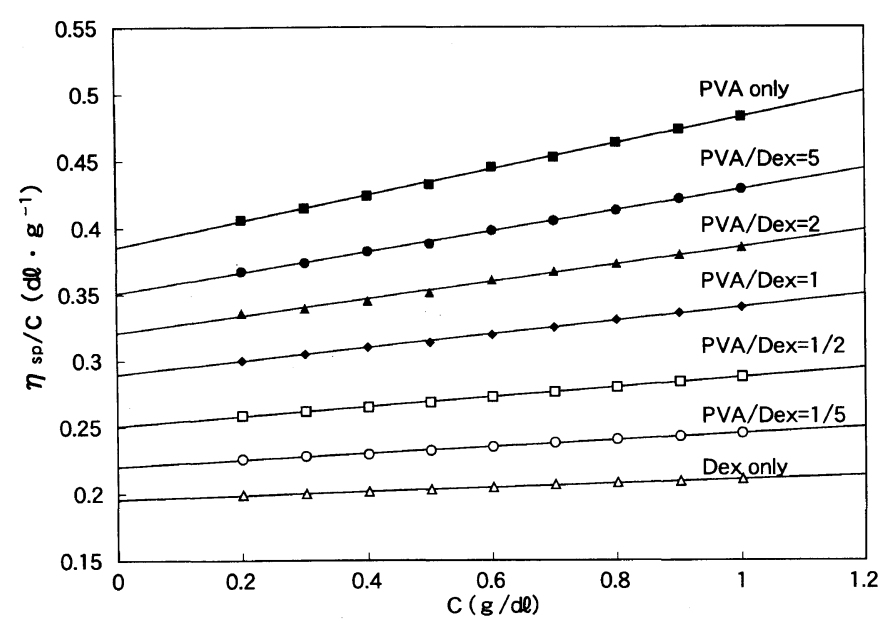

Figure 1. Huggins' plots for the water-Dex(2)-PVA(3) system at $30^{\circ} \mathrm{C}$.

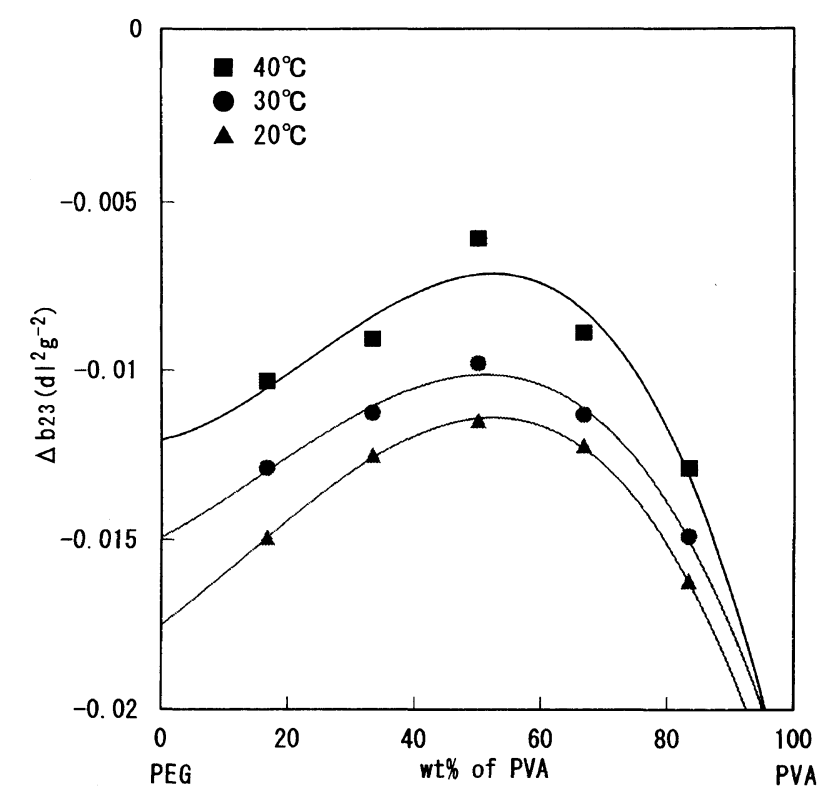

Figure 2. Polymer composition dependence of interactions for the water-PEG-PVA system. interactions between polymer and polymer, or polymer and solvent increased. ${ }^{5}$

Figures 2-10 show the polymer 2-polymer 3 interaction parameters, $\Delta b_{23}$, against polymer content for water-PEG-PVA, water-PVP-PVA, water-Dex-PVA, water-Pul-PVA, water-PVP-PEG, water-Dex-PEG, water-Pul-PEG, water-PVP-Dex, and water-Pul-PVP systems. For only the water-Dex-PVA system, $\Delta b_{23}$ is positive, but for the other systems $\Delta b_{23}$ negative. This indicates that most polymer pairs have repulsive interactions and are incompatible, and the water-Dex-PVA system has attractive interactions and shows compatibility. This seems related to the finding in this study that all water-soluble polymer pairs show liquid-liquid phase separations in water. ${ }^{3}$ There seems to exist domains of polymer 2 or polymer 3 . As the temperature increased, only the water-Dex-PVA system showed

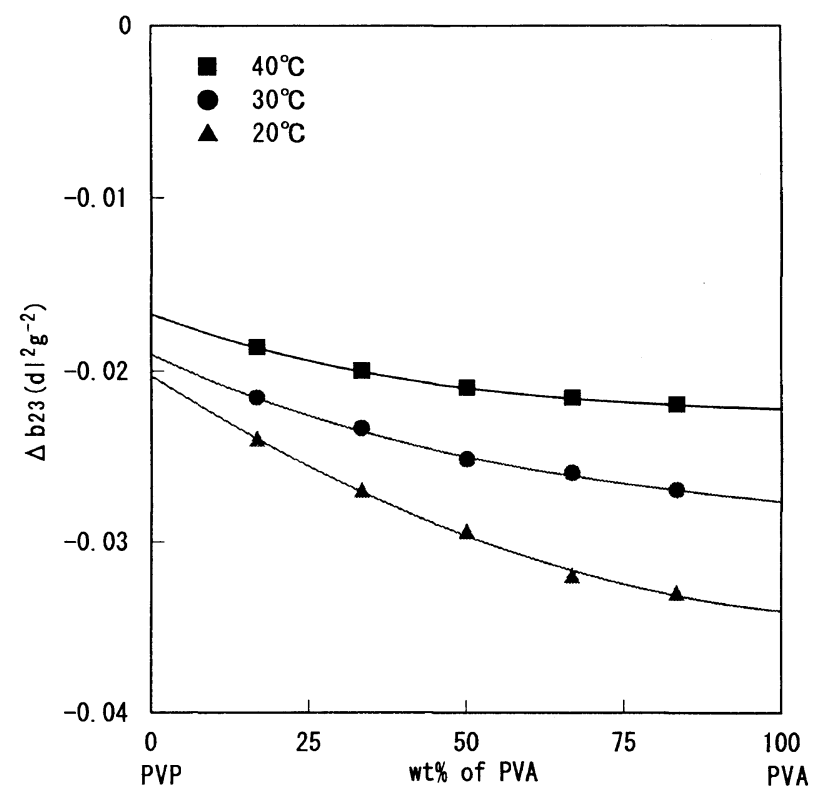

Figure 3. Polymer composition dependence of interactions for the water-PVP-PVA system.

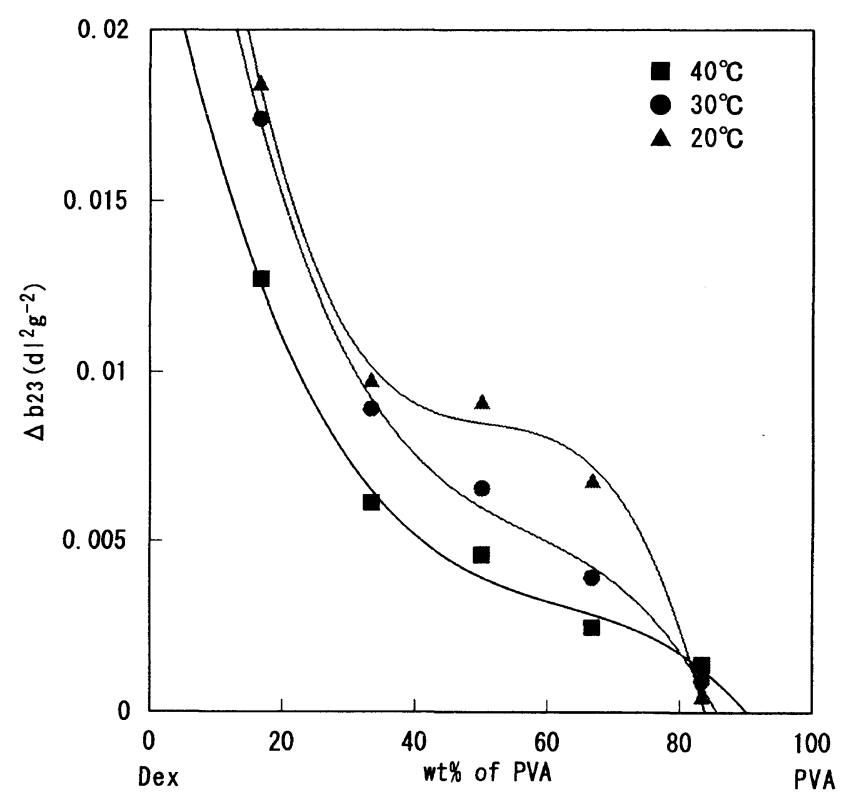

Figure 4. Polymer composition dependence of interactions for the water-Dex-PVA system.

Polym. J., Vol. 29, No. 2, 1997 


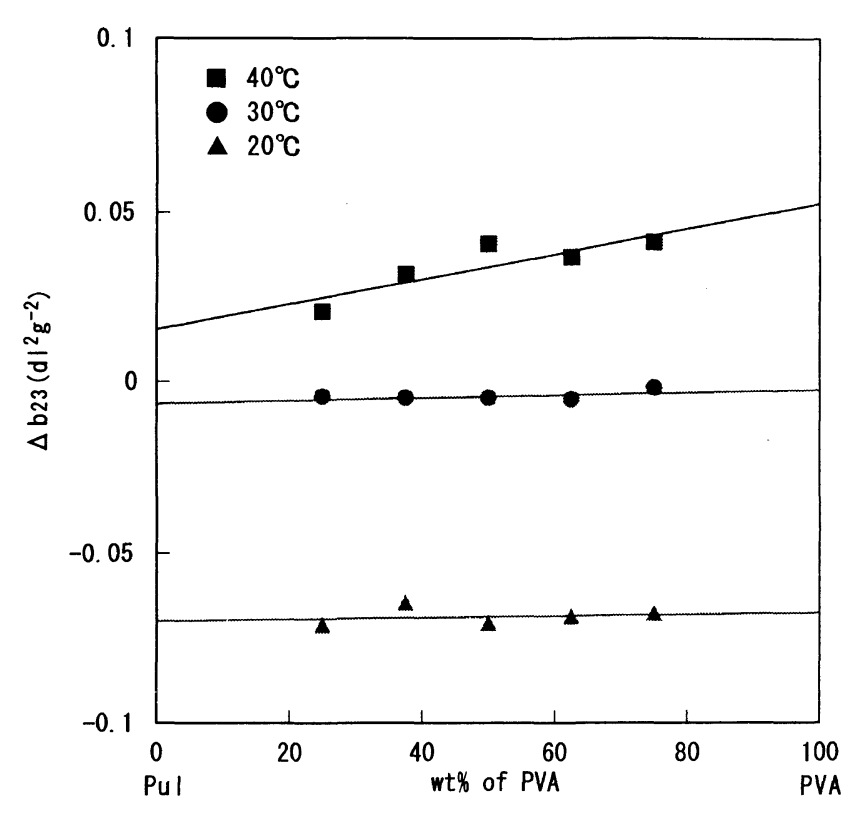

Figure 5. Polymer composition dependence of interactions for the water-Pul-PVA system.

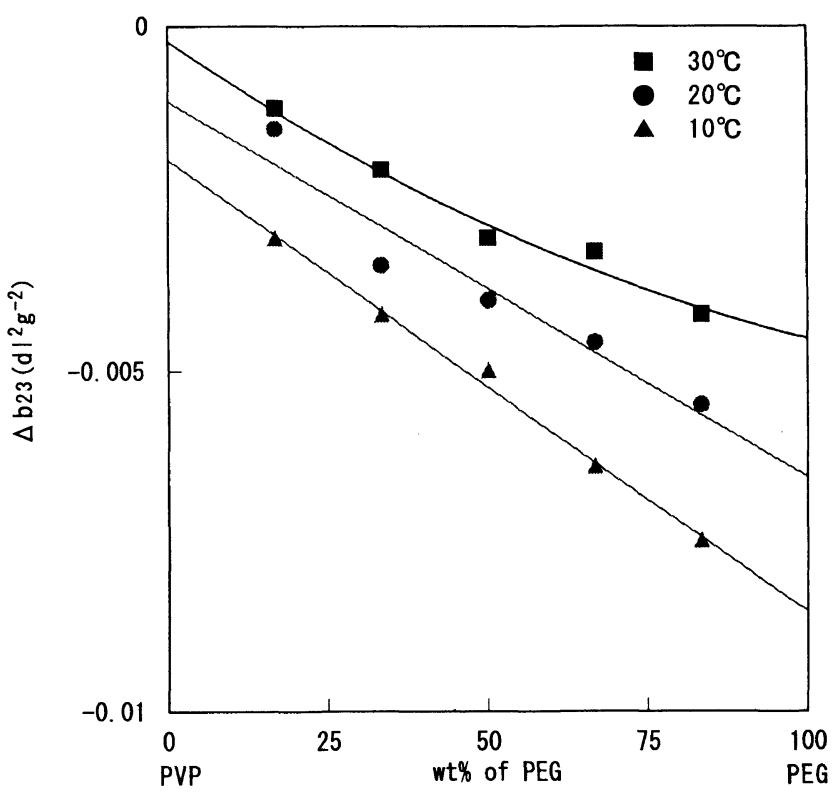

Figure 6. Polymer composition dependence of interactions for the water-PVP-PEG system.

decreased $\Delta b_{23}$, but the other systems showed increased $\Delta b_{23}$. This indicates that for most listed polymer pairs, as the temperature increased, attractive interactions and compatibility were enhanced. Only for the water-DexPVA system, as temperature increased, the repulsive interaction and incompatibility grew. Perhaps, this may be due to more hydrogen bonds between Dex and PVA. ${ }^{6}$ The interactions of hydrogen bonds become weaker as the temperature increases.

Fffects of polymer composition on $\Delta b_{23}$ are stated as follows. For the water-PEG-PVA system as shown in Figure 2, $\Delta b_{23}$ shows the curve with maximum at 50 wt $\%$ of PVA. For the water-PVP-PVA system shown in Figure 3, $\Delta b_{23}$ decreased as wt $\%$ of PVA increased. Therefore, repulsive interactions increase as $\mathrm{wt} \%$ of PVA increases. For the water-Dex-PVA system shown in Figure $4, \Delta b_{23}$ increased as wt $\%$ of Dex increased.

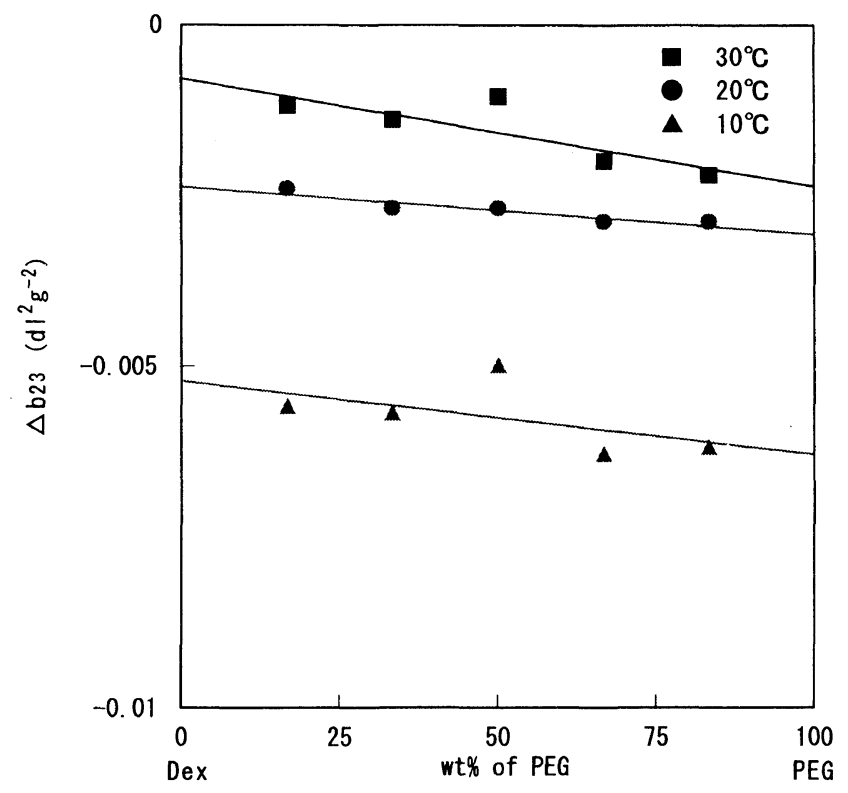

Figure 7. Polymer composition dependence of interactions for the water-Dex-PEG system.

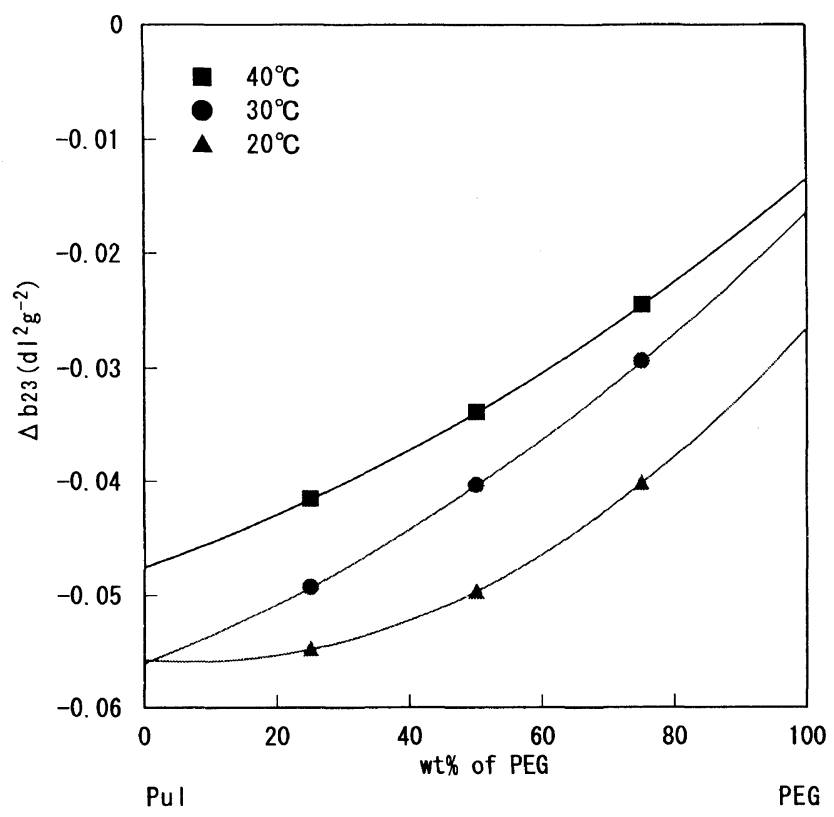

Figure 8. Polymer composition dependence of interactions for the water-Pul-PEG system.

Therefore, attractive interactions increase as $\mathrm{wt} \%$ of Dex increases. For water-Pul-PVA system shown in Figure $5, \Delta b_{23}$ shows a straight line. $\Delta b_{23}$ hardly changed, as $w \mathrm{t} \%$ of PVA increased. For the water-PVP-PEG system (Figure 6), $\Delta b_{23}$ shows a straight line. $\Delta b_{23}$ decreased as wt $\%$ of PEG increased. For the waterDex-PEG system (Figure 7), $\Delta b_{23}$ shows a straight line. $\Delta b_{23}$ decreased as $\mathrm{wt} \%$ of PEG increased. For the water-Pul-PEG system (Figure 8 ), $\Delta b_{23}$ shows a curved line. As shown here, $\Delta b_{23}$ decreased as $\mathrm{wt} \%$ of $\mathrm{Pul}$ increased. For the water-PVP-Dex system (Figure 9), $\Delta b_{23}$ shows a curved line. $\Delta b_{23}$ decreased as wt $\%$ of PVP increased. For the water-Pul-PVP system (Figure 10), $\Delta b_{23}$ shows a curve with a maximum at $50 \mathrm{wt} \%$ of PVP. Figure 2 and Figure 10 show curves with maximum at $50 \mathrm{wt} \%$ of PVA and PVP, respectively. A mixture of 


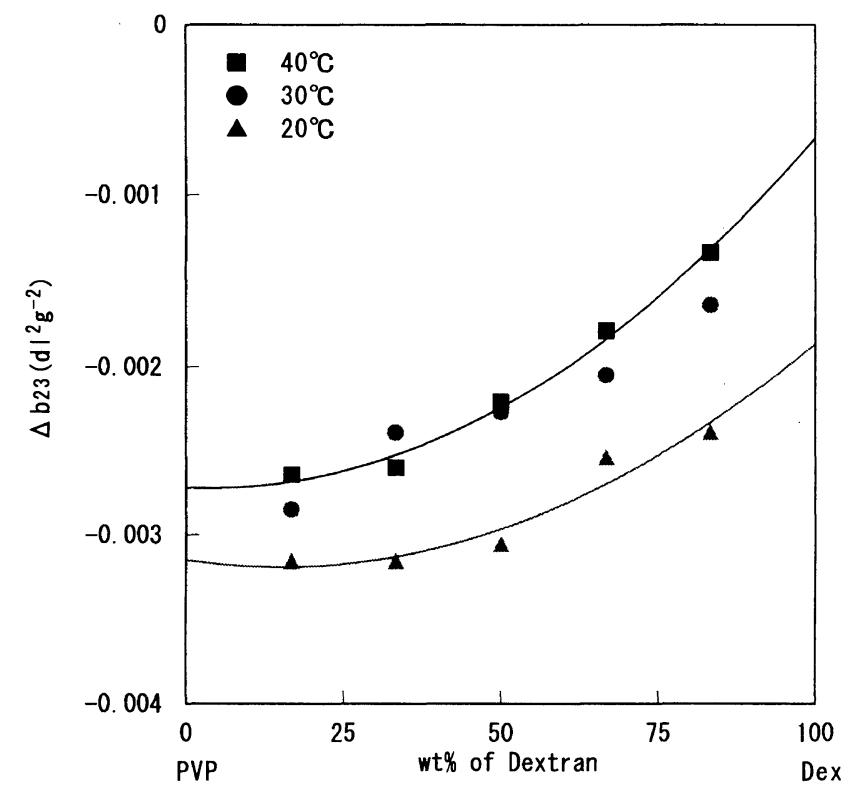

Figure 9. Polymer composition dependence of interactions for the water-PVP-Dex system.

polymers at $1: 1$ enhanced compatibility.

\section{CONCLUSIONS}

For only the water-Dex-PVA system, $\Delta b_{23}$ is positive, whereas for the other systems, negative. This indicates that most polymer pairs have repulsive interactions and show incompatibility.

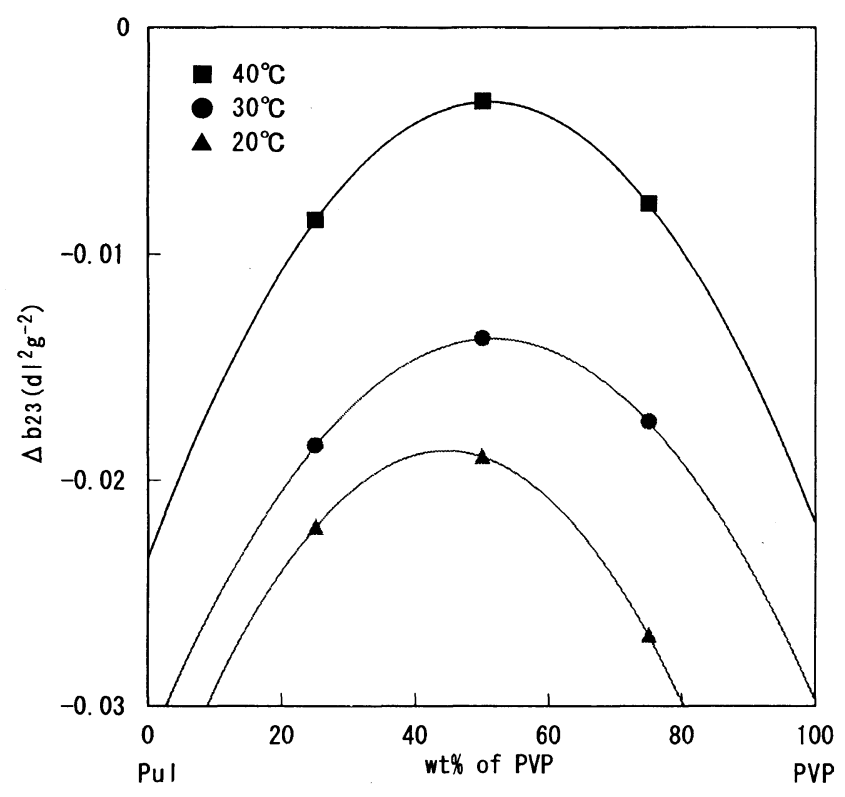

Figure 10. Polymer composition dependence of interactions for the water-Pul-PVP system.

\section{REFERENCES}

1. W. R. Krigbaum and F. T. Wall, J. Polym. Sci., 5, 505 (1950).

2. V. Soria, J. E. Figueruelo, and A. Campos, Eur. Polym. J., 17, 137 (1981).

3. I. Inamura and Y. Jinbo, Polym. J., 23, 1143 (1991).

4. S. Suto, M. Oshima, O. Takatsu, and M. Karasawa, Kobunshi Ronbunshu, 43, 237 (1986).

5. L. H. Cragg and C. C. Bigelow, J. Polym. Sci., 16, 177 (1955).

6. R. J. Hefford, Polymer, 25, 979 (1984). 